\title{
18F-fluorocholine positron emission tomography-computed tomography (18F-FCH PET/CT) for staging of high-risk prostate cancer patients
}

\author{
Simon Gauvin, $\mathrm{MD}^{1}$; Alexis Rompré-Brodeur, $\mathrm{MD}^{2}$; Guillaume Chaussé, $\mathrm{MD}^{3}$; Maurice
} Anidjar, $\mathrm{MD}, \mathrm{PhD}^{2}$; Franck Bladou, $\mathrm{MD}^{2}$; Stephan Probst, $\mathrm{MD}^{3}$

${ }^{1}$ Department of Radiology; ${ }^{2}$ Department of Urology; ${ }^{3}$ Department of Nuclear Medicine; Jewish General Hospital, McGill University Health Centre, Montreal, QC, Canada

Acknowledgements: This work was supported by the Departments of Nuclear Medicine and Urology, Jewish General Hospital, Montreal, QC, Canada.

Cite as: Can Urol Assoc J 2018 September 27; Epub ahead of print. http://dx.doi.org/10.5489/cuaj.5142

Published online September 27, 2018

\section{Abstract}

Introduction: We sought to evaluate the diagnostic performance of 18F-fluorocholine positron emission tomography-computed tomography (18F-FCH PET/CT) for initial staging of patients with high-risk prostate cancer. Secondary objectives were to compare the value of $18 \mathrm{~F}-\mathrm{FCH}$ PET/CT to conventional imaging modalities and to evaluate its clinical impact.

Methods: We conducted a retrospective study including 76 patients who underwent 18F-FCH PET/CT for initial staging of high-risk prostate cancer. Using pre-established validation criteria, sensitivity and specificity were determined for metastatic disease. Results were compared to findings on magnetic resonance imaging (MRI), computed tomography (CT), and bone scan (BS) when available.

Results: Twenty-two (29\%) PET/CT scans were positive, 49 (64\%) negative, and five (7\%) equivocal for nodal or metastatic disease. Of the positive scans, 17 showed regional lymph node involvement, 12 distant nodes, five bone metastases, and three lung metastases. Overall perpatient sensitivity, specificity, positive and negative predictive values for metastatic disease were $65 \%, 100 \%, 100 \%$, and 78\%, respectively. Sensitivity, specificity, positive and negative predictive values were $64 \%, 100 \%, 100 \%$ and $80 \%$, respectively, for nodal involvement and $86 \%, 100 \%, 100 \%$, and $98 \%$ for bone and other metastases. Conventional imaging was negative for the lesion(s) found on PET/CT in five patients. PET/CT changed the clinical management in nine patients $(12 \%)$. 
Conclusions: Although 18F-FCH PET/CT offers some benefits over conventional imaging and demonstrates a high specificity, it remains limited by its sensitivity in the context of high-risk prostate cancer staging. PET with novel urea-based small molecule PSMA inhibitors may overcome some of these limitations. However, the interpretation of the study result is limited by the lack of available histological gold standard, the inclusion of several patients who received androgen-deprivation therapy (ADT) prior to PET/CT, our retrospective design, and a relatively small sample size.

\section{Introduction}

Prostate adenocarcinoma (PCa) is the most common cancer among Canadian men. ${ }^{1}$ Adequate management of newly diagnosed prostate cancer relies primarily on proper staging and assessment of risk-group stratification. While most of prostate cancer cases are of very-low, low and intermediate-risk category, high-risk prostate cancer represents between $16 \%-31 \%$ of cases at time of diagnosis depending on the classification system used. ${ }^{2,3}$ Notably, the incidence of high-risk localized and metastatic prostate cancer seems to be increasing. ${ }^{4}$ Treatment options for high-risk localized prostate cancer include radical prostatectomy (RP) with pelvic lymph node dissection (PLND) or external beam radiation therapy (EBRT) with the addition of androgen deprivation therapy (ADT). ${ }^{5}$ If the prostate cancer is metastatic at diagnosis, especially in a highvolume context, it is best managed with systemic therapies such as ADT alone or in combination with chemotherapy or abiraterone. ${ }^{6-8}$

Given the higher likelihood of metastatic disease in the high-risk patients as well as the different available treatment options, accurate detection of nodal or skeletal disease is crucial in this group. For this reason, imaging plays a key role in prostate cancer staging. However, the optimal imaging modality remains an ongoing debate. Most clinicians use a combination of magnetic resonance imaging (MRI), computed tomography (CT) and bone scan (BS) to perform staging despite poor reported sensitivity and specificity. ${ }^{9}$

In recent years, nuclear medicine imaging markers have been developed in an attempt to overcome this gap, such as ${ }^{11} \mathrm{C}$-Choline and ${ }^{18} \mathrm{~F}$-fluorocholine $(18 \mathrm{~F}-\mathrm{FCH}) .{ }^{10}$ Choline plays an essential role in the formation of phospholipid membranes and demonstrates increased uptake in prostate cancer cells. ${ }^{11}$ The role of $18 \mathrm{~F}-\mathrm{FCH}$ positron emission tomography-computed tomography (PET/CT) in prostate cancer has previously been studied for initial staging and in context of biochemical recurrence with mixed results. ${ }^{12-16}$ In studies looking at the potential role of 18F-FCH PET/CT in initial prostate cancer staging, the benefits seemed to be optimal when investigating high-risk prostate cancer, but the number of patients included in the studies was small and both intermediate and high-risk patients were often analyzed together, which limited extrapolation of results. ${ }^{13,15,17}$

The primary objective of this study was to evaluate the diagnostic performance of $18 \mathrm{~F}$ FCH PET/CT for detection of metastatic disease in high-risk prostate cancer patients at the time of initial staging. Secondary objectives were to compare its value to conventional imaging (MRI, CT and BS) and to evaluate its clinical impact. 


\section{Methods}

Patients and study design

We conducted a single-center retrospective study including 76 patients who underwent 18F-FCH PET/CT scans performed from March 2014 to March 2017. Study protocols were approved by the hospital research ethics committee (REB \#13-047 and \#16-061). Health Canada approval and written informed consent from all patients were obtained. Inclusion criteria consisted of: histologically proven diagnosis of prostate adenocarcinoma with high-risk features as defined by any one of: prostate-specific antigen (PSA) level $>20 \mathrm{ng} / \mathrm{mL}$, Gleason score $\geq 8$ or clinical $\mathrm{T}$ stage (cT) $\geq \mathrm{T} 3$ a based on digital rectal examination. ${ }^{18}$ Exclusion criteria consisted of: any previously known metastasis or previous treatment for prostate cancer (other than ADT preceding PET/CT in view of a combined treatment approach after imaging), any known concurrent active cancer(s) and technically non-diagnostic study (Figure 1). Patient characteristics are presented in Table 1.

Technique and study interpretation

PET/CT preparation consisted of 4 h fasting. Approximately $4 \mathrm{MBq} / \mathrm{kg}$ IV of $18 \mathrm{~F}-$ fluoromethylcholine were administered. Examinations were performed using a hybrid PET/CT scanner (GE Discovery ST, General Electric Medical Systems, Waukesha, WI, USA). Images were acquired 10-30 min post-injection from skull base to mid-thighs (6-7 bed positions, 4 $\mathrm{min} / \mathrm{bed}$ ). A nuclear medicine specialist interpreted the studies as positive, negative or equivocal; a positive examination was defined as $18 \mathrm{~F}-\mathrm{FCH}$ uptake higher than surrounding background activity and not explained by physiological process.

\section{Validation of results}

PET/CT findings were compared to MRI, CT, bone scan, and histological analysis when available. Results were considered true positive if meeting any of the following validation criteria: 1) positive histological analysis, 2) treatment response on follow-up imaging, 3) progression on follow-up imaging or 4) positive conventional imaging at initial staging. Results were considered true negative if there was: 1 ) negative histological analysis or 2) negative clinical and/or imaging follow-up for at least 6 months following PET/CT. A negative clinical follow-up was defined as no increase in PSA value or clinical evidence of metastatic disease during the follow-up period. Results were considered false positive if there was: 1) negative histological analysis or 2) imaging follow-up demonstrating either stable initial findings for at least 6 months or improved findings any time after PET/CT without treatment. Results were considered false negative if there was: 1) positive histological analysis, 2) treatment response on imaging any time after PET/CT, 3) positive conventional imaging at initial staging or 4) clinical and/or imaging evidence of metastatic disease detected within 6 months following PET/CT. Sensitivity, specificity, positive and negative predictive values were then calculated on a perpatient basis.

\section{Clinical management}


A summary of clinical information was provided to a senior uro-oncologist with more than 20 years of experience in prostate cancer management who was blinded to patient identifying information, PET/CT results and information available afterwards. The uro-oncologist was asked to determine the theoretical management had the PET/CT not been performed. The theoretical and actual therapeutic strategies were then compared.

Statistical analysis

All statistical analyses were conducted using the software R (v.3.4.1) ${ }^{19}$ For descriptive statistics, we computed t-test for continuous variables or performed Pearson's Chi-squared test for categorical variables. Results with $p<0.05$ were considered statistically significant. Finally, we evaluated inter-agreement between PET/CT and conventional imaging results using Cohen's kappa coefficient.

\section{Results}

PET/CT results and location of disease

Seventy-six patients underwent PET/CT for initial staging of high-risk prostate cancer with a median age of 67.8 years. Seventy-three (96\%) PET/CT scans were positive, one (1\%) negative and 2 (3\%) equivocal for prostate lesion(s). Twenty-two (29\%) PET/CT scans were positive, 49 (64\%) negative and 5 (7\%) equivocal for metastatic disease. Of the 22 positive scans, 17 (77\%) demonstrated regional nodal involvement, 12 (55\%) distant nodal involvement, 5 (23\%) bone metastasis and 3 (14\%) lung metastasis. Examples of positive PET/CT scans are presented in Figures 2, 3 and 4.

\section{Diagnostic performance and validation of PET/CT results}

Excluding equivocal results $(\mathrm{n}=4)$ and cases where pre-established validation criteria could not be applied due to absent follow-up information ( $\mathrm{n}=15)$, overall sensitivity, specificity, positive and negative predictive values for nodal or metastatic disease were $65 \%, 100 \%, 100 \%$ and $78 \%$, respectively. Seventeen of 22 positive PET/CT scans for metastatic disease (77\%) were validated according to treatment response on follow-up imaging in 12 patients (70\%), histological analysis in 3 patients (18\%), progression of lesion in one patient (6\%) and correlation with conventional imaging at initial staging in one patient (6\%).

Excluding equivocal results $(\mathrm{n}=2)$ and cases missing necessary information to validate results ( $\mathrm{n}=14)$, sensitivity, specificity, positive and negative predictive values were $64 \%, 100 \%$, $100 \%$ and $80 \%$, respectively for detection of nodal metastases. Sixteen of 21 positive PET/CT scans for regional and/or non-regional lymph nodes (76\%) were validated according to treatment response on follow-up imaging in 11 patients (69\%), histological analysis in 3 patients (19\%) and correlation with conventional imaging at initial staging in 2 patients (12\%). Five PET/CT scans positive for nodal metastasis could not be validated due to lack of available information.

Looking more specifically at the subgroup of patients who underwent RP with PLND ( $n=26$ ), we found a sensitivity, specificity, positive and negative predictive values of $10 \%, 100 \%$, $100 \%$ and $64 \%$, respectively for regional lymph node metastases based on histological analysis. 
Among these patients, 9 had false negative PET/CT results for regional lymph node metastases; 6 of these had macrometastases $(>2 \mathrm{~mm})$ and $3 \mathrm{had}$ micrometastases $(\leq 2 \mathrm{~mm})$, as classified on histopathology.

Excluding equivocal results $(\mathrm{n}=2)$ and cases missing necessary information to validate results ( $\mathrm{n}=11)$, sensitivity, specificity, positive and negative predictive values for distant metastases were $86 \%, 100 \%, 100 \%$ and $98 \%$, respectively. All PET/CT scans positive for bone and lung metastases $(n=6)$ were validated according to treatment response in 4 patients (67\%) and progression of findings on follow-up imaging studies in 2 patients (33\%).

\section{Comparison with conventional imaging modalities}

Among the 21 PET/CT scans showing lymph node involvement, conventional imaging was performed in 18 patients (86\%). Of these, conventional imaging demonstrated the lesion(s) found on PET/CT in 11 patients (61\%), was negative in 5 patients (28\%) and was indeterminate in 2 patients (11\%). Excluding indeterminate cases on conventional imaging, PET/CT and conventional imaging were both positive in 11 cases and both negative in 43 cases (good agreement, $\kappa=0.68, \mathrm{p}<0.001)$. Conventional imaging was falsely positive in one patient and falsely negative in 10 patients.

Among the 5 PET/CT scans showing bone involvement, bone scan was performed in 4 patients (80\%). Of these, bone scan demonstrated the lesion(s) found on PET/CT in one patient (25\%), was negative in 2 patients (50\%) and was indeterminate in one patient (25\%). Excluding indeterminate cases on bone scan, PET/CT and bone scan were both positive in one case and both negative in 42 cases (fair agreement, $\kappa=0.29, \mathrm{p}=0.048$ ). Bone scan was falsely positive in 2 patients and falsely negative in 3 patients.

Association between patient characteristics and PET/CT results

When comparing positive and negative PET/CT scans for metastatic disease, there were statistically significant differences depending on clinical $\mathrm{T}$ and $\mathrm{N}$ stages as well as in Gleason scores (Table 2). Of note, 18 of 22 PET/CT scans positive for metastatic disease were found in patients with a Gleason score of 9. Moreover, all 5 patients whose PET/CT demonstrated abnormal regional lymph node(s) despite negative initial conventional imaging (cN0) also had a Gleason score of 9. Patients with metastatic disease on PET/CT were more likely to have a higher proportion of positive cores on initial biopsy (75\% vs 56\%, p=0.01), therefore reflecting higher prostate volume disease. Finally, although we observed a trend for higher PSA levels in patients with positive PET/CT scans, this did not reach statistical significance (mean PSA 64.1 vs $24.8 \mathrm{ng} / \mathrm{mL}, \mathrm{p}=0.09)$.

\section{Comparison between theoretical and actual clinical management strategies}

The theoretical and actual management strategies differed in 9 patients (12\%). In 5 cases, pelvic EBRT was added to ADT for treatment of locoregional disease after PET/CT excluded distant metastasis. In 3 cases, surgery was preferred after PET/CT clarified indeterminate results on CT and/or bone scan. Finally, in one case, stereotactic body radiation therapy (SBRT) was added to pelvic EBRT for treatment of oligometastatic skeletal disease. 


\section{Discussion}

In this study, we report our initial Canadian single-center experience with 18F-FCH PET/CT in the context of initial staging of high-risk prostate cancer.

The value of 18F-FCH PET/CT in initial staging of intermediate to high-risk of prostate cancer is controversial with previous studies demonstrating low sensitivity and high

specificity. ${ }^{12,15,17,20}$ In a meta-analysis including 441 patients studying the role of 11C-Choline and 18F-FCH, Evangelista et al. found a pooled sensitivity and specificity of $49.2 \%$ (95\% CI, 39.9-58.4) and 95\% (95\% CI, 92-97.1) for nodal involvement on a per-patient basis. ${ }^{20}$ In a study including 132 patients with intermediate to high-risk prostate cancer, Beheshti et al. reported a sensitivity, specificity, positive and negative predictive values of 45\%, 96\%, 82\% and 83\% for malignant lymph nodes on per-patient analysis. ${ }^{12}$ Similar to previous studies, we found a sensitivity, specificity, positive predictive and negative predictive values of $64 \%, 100 \%, 100 \%$ and $80 \%$ for metastatic disease to lymph nodes. Unlike other previous studies, we included only patients with high-risk prostate cancer, which could partly explain the higher positive predictive value obtained. In the subgroup of patients who underwent RP with PLND and for which histological analysis was available ( $n=26)$, we found that PET/CT had a low sensitivity of $10 \%$ and high specificity of $100 \%$ for regional lymph node metastases, with a high rate of false negative results (9 patients). This is consistent with Kjölhede et al. who found that $18 \mathrm{~F}-\mathrm{FCH}$ PET/CT had a low sensitivity of 33\% and high specificity of 92\% in a study including 112 patients with extensive PLND. ${ }^{16}$ Finally, as previously reported, we also found a higher sensitivity for bone and other metastases (86\%) than for nodal metastases (64\%). ${ }^{13}$

Interestingly, the majority of patients with a PET/CT positive for metastatic disease (18/22 patients, 82\%) had a Gleason score of 9 as did all 5 patients whose PET/CT demonstrated abnormal regional lymph node(s) despite negative initial conventional imaging (cN0). Therefore, we could hypothesize that perhaps the greatest utility of 18F-FCH PET/CT at detecting metastatic disease during initial staging lies in patients with Gleason scores $\geq 9$. Nevertheless, interpretation of the results remains limited by the fact that a multivariate analysis was not performed due to our relatively small sample size. Other patient factors which are integral to the initial clinical risk stratification (clinical staging and PSA) must be considered as they may also likely contribute to PET/CT positivity.

18F-FCH PET/CT detected metastatic disease not otherwise identified by conventional imaging in 5/18 cases of nodal involvement (28\%) and 2/4 cases of bone metastases (50\%). These results are consistent with Evangelista et al. who established that 18F-FCH PET/CT had a higher sensitivity than CT (69.2\% vs 46.2\%) and bone scan (100\% vs 90\%) for detection of metastatic disease. $^{13}$

In our study, we determined that the theoretical retrospective management strategy differed from the actual prospective management strategy in 9 patients (12\%). This result is in agreement with previous studies showing a change in the therapeutic option in $5-20 \%$ of cases using 18F-FCH PET/CT for staging of intermediate to high-risk prostate cancer. ${ }^{12,13,21,22}$ 
Since the introduction of 18F-FCH PET/CT, more sensitive and specific PET tracers have been developed to increase the yield of PET/CT at initial staging and for evaluation of biochemical recurrence. In a study including 130 patients with intermediate to high-risk prostate cancer who underwent RP with PLND, Maurer et al. found that ${ }^{68}$ Ga-PSMA-PET/CT had a sensitivity of $65.9 \%$ and specificity of $98.9 \%$ for detection of lymph node metastases on a perpatient basis. ${ }^{23}$ Similarly, in a smaller study including 34 patients who underwent ${ }^{68}$ Ga-PSMAPET/CT for nodal staging prior to RP with primary PLND or secondary PLND, Herlemann et al. obtained a sensitivity of $84 \%$ and specificity of $82 \%{ }^{24}$ Although $18 \mathrm{~F}-\mathrm{FCH}$ PET/CT offers some benefits over conventional imaging and demonstrates a high specificity, it remains limited by its sensitivity in the context of high-risk prostate cancer staging. For this reason, we suggest that future research be done with novel tracers such as ${ }^{68} \mathrm{Ga}$-PSMA as it may overcome some of the limitations encountered.

There are several limitations to our study. The main limitation consists of the lack of histological confirmation available for detected metastatic lesions with PET/CT in a total of 19 patients (25\%). This limitation is particularly emphasized when looking at patients who underwent non-surgical management such as EBRT or chemotherapy where histological confirmation of the metastatic lesions seen was not available in 17 patients (22\%). This also applies to patients whose PET/CT revealed distant nodal (10 patients, 13\%) and osseous metastatic lesions (6 patients, 8\%) for which biopsy is rarely clinically justified or performed. Therefore, we opted for a set of pre-defined validation criteria based on response to treatment or progression of disease on subsequent imaging and comparison with findings on conventional imaging modalities, as done in previous studies evaluating the role of 18F-FCH PET/CT in staging of prostate cancer. ${ }^{12,13}$

Another limitation of our study consists of the inclusion of a few patients who received ADT prior to PET/CT. While the nuclear medicine specialist who interpreted the PET/CT studies was not blinded to the ADT status of patients scanned, this could have led to error in the interpretation of 18F-FCH PET/CT. Nine patients (12\%) received ADT before PET/CT; among those, there were 3 false negative results (one bone and 2 regional lymph node metastases). As ADT is known to decrease fluorocholine uptake in sites of hormone-sensitive disease, this may have contributed to the overall false negative rate. ${ }^{25,26}$ Therefore, we suggest that $18 \mathrm{~F}-\mathrm{FCH}$ PET/CT done for staging of high-risk prostate cancer should be avoided in men who previously received $\mathrm{ADT}$. It is important to clarify however that $\mathrm{ADT}$ is not considered a contraindication to 18F-FCH PET/CT in the evaluation of castration-resistant prostate cancer (CRPC) as 18F-FCH $\mathrm{PET} / \mathrm{CT}$ has shown some utility in mapping resistant disease and subsequently assessing treatment efficacy. ${ }^{27,28}$

Finally, our study remains limited by its retrospective design, incomplete patient followup information and relatively small sample size. Nevertheless, to the best of our knowledge, our study represents one of the largest single cohorts of patients with high-risk prostate cancer studied with 18F-FCH PET/CT. 


\section{Conclusion}

In conclusion, although 18F-FCH PET/CT offers some benefits over conventional imaging and demonstrates a high specificity, it remains limited by its sensitivity in the context of high-risk prostate cancer staging. PET with novel urea-based small molecule PSMA inhibitors may overcome some of these limitations. 


\section{References}

1. Cancer.ca. Canadian Cancer Society’s Advisory Committee on Cancer Statistics; 2017 [updated 2017; cited 2018 May 19]. http://www.cancer.ca/. Accessed May 19, 2018.

2. D'Amico AV, Whittington R, Malkowicz SB, et al. Biochemical outcome after radical prostatectomy, external beam radiation therapy, or interstitial radiation therapy for clinically localized prostate cancer. JAMA 1998;280:969-74.

3. Cooperberg MR, Cowan J, Broering JM, et al. High-risk prostate cancer in the United States, 1990-2007. World J Urol 2008;26:211-8.

4. Weiner AB, Matulewicz RS, Eggener SE, et al. Increasing incidence of metastatic prostate cancer in the United States (2004-2013). Prostate Cancer Prostatic Dis 2016;19:395-97.

5. Sanda MG, Chen RC, Crispino T, et al. 2017 [cited 2017 November 24]. http://www.auanet.org/guidelines/clinically-localized-prostate-cancer-new-(aua/astro/suoguideline-2017). Accessed November 24, 2017.

6. Sweeney CJ, Chen YH, Carducci M, et al. Chemohormonal therapy in metastatic hormonesensitive prostate cancer. $N$ Engl J Med 2015;373:737-46.

7. James ND, de Bono JS, Spears MR, et al. Abiraterone for prostate cancer not previously treated with hormone therapy. N Engl J Med 2017;377:338-51.

8. Fizazi K, Tran N, Fein L, et al. Abiraterone plus prednisone in metastatic, castrationsensitive prostate cancer. N Engl J Med 2017;377:352-60.

9. Crawford ED, Stone NN, Yu EY, et al. Challenges and recommendations for early identification of metastatic disease in prostate cancer. Urology 2014;83:664-9.

10. Vali R, Loidl W, Pirich C, et al. Imaging of prostate cancer with PET/CT using (18)FFluorocholine. Am J Nucl Med Mol Imaging 2015;5:96-108.

11. Ramirez de Molina A, Rodriguez-Gonzalez A, Gutierrez R, et al. Overexpression of choline kinase is a frequent feature in human tumor-derived cell lines and in lung, prostate, and colorectal human cancers. Biochem Biophys Res Commun 2002;296:580-3.

12. Beheshti M, Imamovic L, Broinger G, et al. 18F choline PET/CT in the preoperative staging of prostate cancer in patients with intermediate or high risk of extracapsular disease: a prospective study of 130 patients. Radiology 2010;254:925-33.

13. Evangelista L, Cimitan M, Zattoni F, et al. Comparison between conventional imaging (abdominal-pelvic computed tomography and bone scan) and [(18)F]choline positron emission tomography/computed tomography imaging for the initial staging of patients with intermediate- tohigh-risk prostate cancer: a retrospective analysis. Scand J Urol 2015;49:345-53.

14. "AUTHOR", 2017.

15. Hacker A, Jeschke S, Leeb K, et al. Detection of pelvic lymph node metastases in patients with clinically localized prostate cancer: comparison of [18F]fluorocholine positron emission tomography-computerized tomography and laparoscopic radioisotope guided sentinel lymph node dissection. J Urol 2006;176:2014-8; discussion 18-9.

16. Kjolhede H, Ahlgren $\mathrm{G}$, Almquist $\mathrm{H}$, et al. (1)(8)F-fluorocholine PET/CT compared with extended pelvic lymph node dissection in high-risk prostate cancer. World J Urol 2014;32:965-70.

17. Umbehr MH, Muntener M, Hany T, et al. The role of $11 \mathrm{C}$-choline and $18 \mathrm{~F}$-fluorocholine positron emission tomography (PET) and PET/CT in prostate cancer: a systematic review and meta-analysis. Eur Urol 2013;64:106-17. 
18. Mohler JL, Armstrong AJ, Bahnson RR, et al. Prostate cancer, version 1.2016. J Natl Compr Canc Netw 2016;14:19-30.

19. Team RC. Vienna, Austria: R Core Team; 2017 [cited 2017 November 24]. https://www.Rproject.org/. Accessed November 24, 2017.

20. Evangelista L, Guttilla A, Zattoni F, et al. Utility of choline positron emission tomography/computed tomography for lymph node involvement identification in intermediate- to high-risk prostate cancer: a systematic literature review and meta-analysis. Eur Urol 2013;63:1040-8.

21. Beheshti M, Vali R, Waldenberger P, et al. Detection of bone metastases in patients with prostate cancer by $18 \mathrm{~F}$ fluorocholine and $18 \mathrm{~F}$ fluoride PET-CT: a comparative study. Eur $J$ Nucl Med Mol Imaging 2008;35:1766-74.

22. Kjolhede H, Ahlgren G, Almquist H, et al. Combined 18F-fluorocholine and 18F-fluoride positron emission tomography/computed tomography imaging for staging of high-risk prostate cancer. BJU Int 2012;110:1501-6.

23. Maurer T, Gschwend JE, Rauscher I, et al. Diagnostic efficacy of (68)Gallium-PSMA positron emission tomography compared to conventional imaging for lymph node staging of 130 consecutive patients with intermediate to high risk prostate cancer. $J$ Urol 2016;195:1436-43.

24. Herlemann A, Wenter V, Kretschmer A, et al. 68Ga-PSMA positron emission tomography/computed tomography provides accurate staging of lymph node regions prior to lymph node dissection in patients with prostate cancer. Eur Urol 2016;70:553-57.

25. Evangelista L, Zattoni F, Guttilla A, et al. The effects of androgen deprivation therapy on the $18 \mathrm{~F}$-choline uptake in prostate cancer patients undergoing neoadjuvant treatment. $Q J$ Nucl Med Mol Imaging 2016.

26. Giovacchini G, Picchio M, Coradeschi E, et al. [(11)C]choline uptake with PET/CT for the initial diagnosis of prostate cancer: relation to PSA levels, tumour stage and antiandrogenic therapy. Eur J Nucl Med Mol Imaging 2008;35:1065-73.

27. Lee J, Sato MM, Coel MN, et al. Prediction of PSA Progression in Castration-Resistant Prostate Cancer Based on Treatment-Associated Change in Tumor Burden Quantified by 18F-Fluorocholine PET/CT. J Nucl Med 2016;57:1058-64.

28. De Giorgi U, Caroli P, Burgio SL, et al. Early outcome prediction on 18F-fluorocholine $\mathrm{PET} / \mathrm{CT}$ in metastatic castration-resistant prostate cancer patients treated with abiraterone. Oncotarget 2014;5:12448-58. 


\section{Figures and Tables}

Fig. 1. Flow diagram outlining inclusion and exclusion criteria and study design. 18F-FCH: 18Ffluorocholine; PET/CT: positron-emission tomography-computed tomography; MRI: magnetic resonance imaging; PSA: prostate-specific antigen.

Patients with 18F-FCH PET/CT performed between

March 26, 2014 and March 9, 2017 (n=254)

Patients excluded on basis of indication or histology $(\mathrm{n}=175)$ :

- $\quad$ Biochemical recurrence $(n=111)$

- $\quad$ Restaging after initial treatment $(n=51)$

- Initial staging of intermediate-risk disease $(n=11)$

- Initial staging of low-risk disease $(n=1)$

- Suspicious prostate lesion on MRI and elevated PSA but not histologically proven prostate cancer $(n=1)$

Indication consisting of initial staging of high or very high-risk disease $(n=79)$

Technically non-diagnostic study (interstitial injection) $(n=1)$

Other active concurrent cancer(s) known at time of PET/CT (n=2)

Patients included in retrospective study $(\mathrm{n}=76)$ 
Fig. 2. Example of local disease on 18F-FCH PET/CT with MRI correlation. (A) Axial 18F-FCH PET/CT images obtained in a 63-year-old man with PCa (cT1c, Gleason score 8, PSA 12.6 $\mathrm{ng} / \mathrm{mL}$ ) showing bilateral prostate uptake (SUV 6.6) (arrows) without metastatic disease. (B) Corresponding axial MRI prostate images (from left to right: T2-weighted images [T2WI], diffusion-weighted imaging [DWI], apparent diffusion coefficient [ADC] map and dynamic contrast-enhanced images [DCE]) demonstrating a non-circumscribed homogeneous moderately T2 hypointense lesion measuring $1.8 \mathrm{~cm}$ in maximal dimension located in the transition zone at the apex and mid-gland with mild extension to the right anterolateral peripheral zone (arrows). There is associated restricted diffusion on DWI/ADC and early focal enhancement on DCE (PIRADS 5). The patient underwent RP with extended PLND (pT3a pN0), without evidence of biochemical recurrence after 10 months of followup.

A.

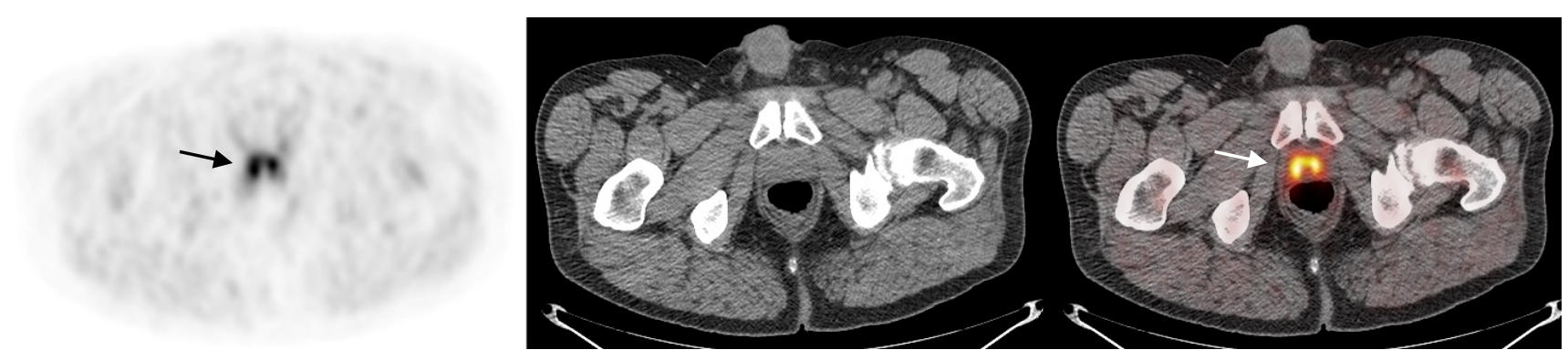

B.

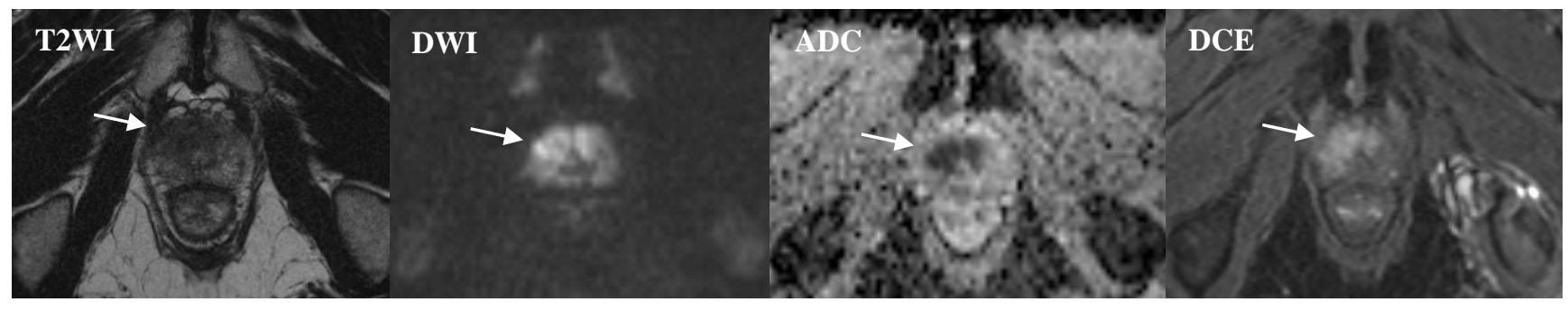


Fig. 3. Examples of regional and distant lymph node involvement on 18F-FCH PET/CT. (A) Axial 18F-FCH PET/CT images obtained in a 68-year-old man with PCa (cT1a, Gleason score 9, PSA $17.2 \mathrm{ng} / \mathrm{mL}$ ) showing intense $18 \mathrm{~F}-\mathrm{FCH}$ uptake (SUV 6.7) in a $1.1 \mathrm{~cm}$ right obturator lymph node (arrows). The patient underwent RP with PLND (pT3a pN1). (B) Axial 18F-FCH PET/CT images obtained in a 78-year-old man with metastatic PCa (cT3b, Gleason score 9, PSA 11.1 $\mathrm{ng} / \mathrm{mL}$ ) showing abnormal uptake in a subcarinal lymph node (arrows), proven to represent PCa metastasis on endobronchial ultrasound-guided transbronchial needle aspiration biopsy (EBUSTBNA).

A.
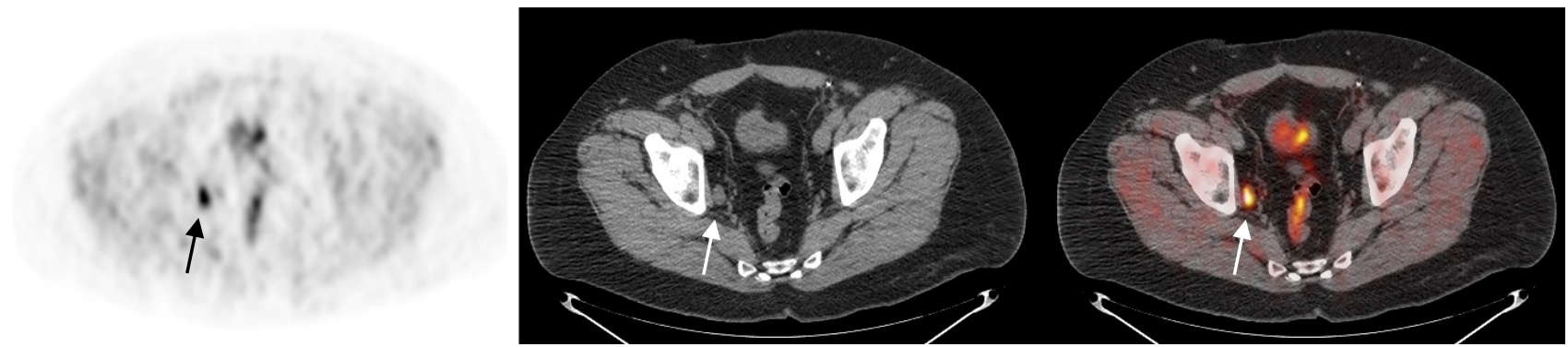

B.

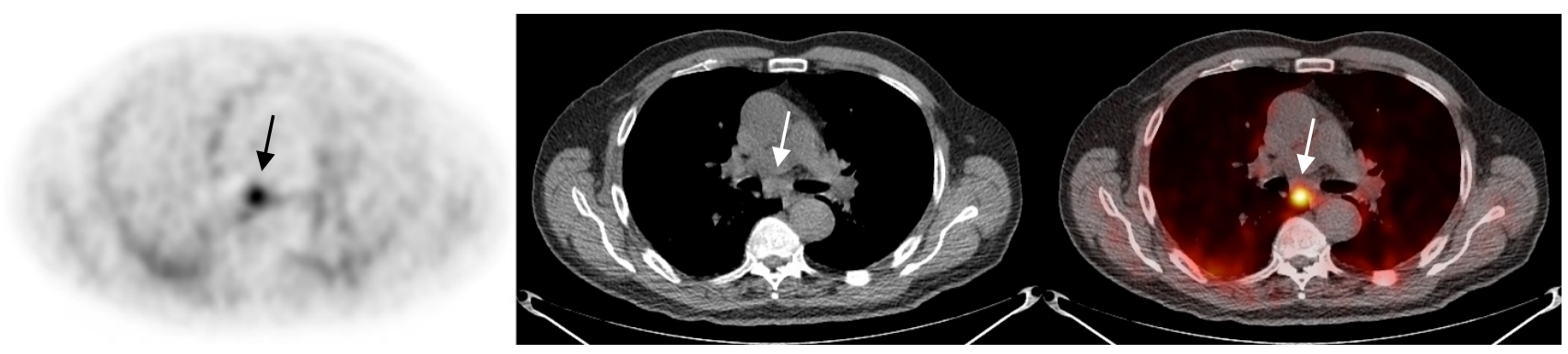


Fig. 4. Example of bone metastasis on 18F-FCH PET/CT. (A) Axial 18F-FCH PET/CT images obtained at initial staging in a 82-year-old man with PCa (cT3b, Gleason score 6, PSA 81.0 $\mathrm{ng} / \mathrm{mL}$ ) showing focal intense manubrial 18F-FCH uptake (SUV 11.8) (arrows). (B) Followup PET/CT obtained 4 months after ADT demonstrates complete metabolic response of the manubrial lesion, which is now densely sclerotic (arrows).

A.

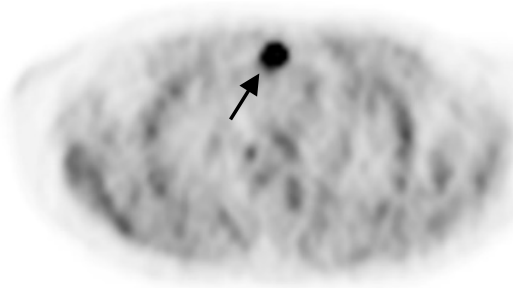

B.

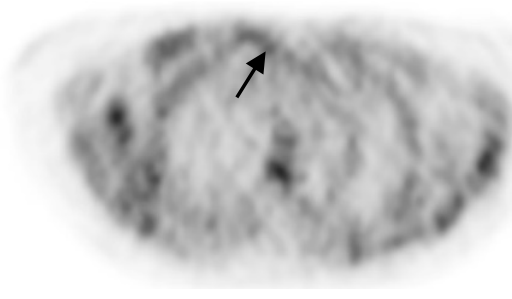

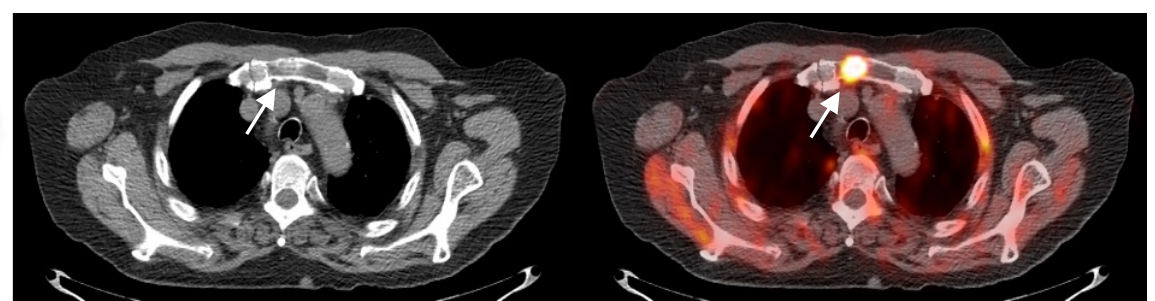

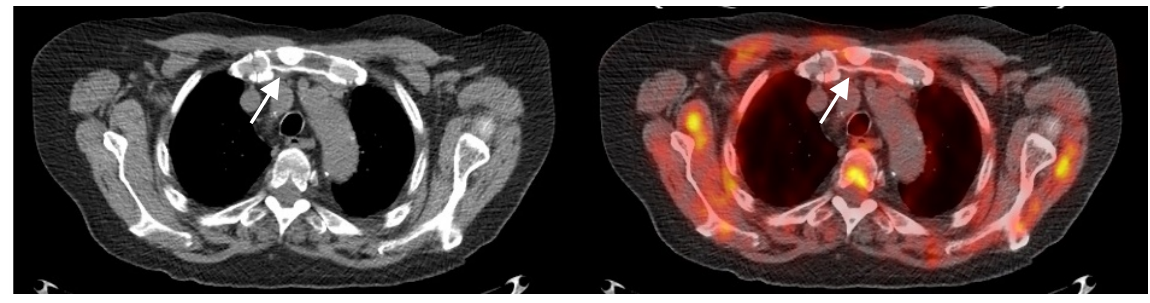




\begin{tabular}{|c|c|}
\hline Characteristics & Value \\
\hline \multicolumn{2}{|l|}{ Age, years } \\
\hline Mean (SD) & $66.5(8.0)$ \\
\hline Median & 67.8 \\
\hline Range & $47.1-82.8$ \\
\hline \multicolumn{2}{|c|}{ PSA at time of PET/CT, ng/mL } \\
\hline Mean (SD) & $36.2(86.3)$ \\
\hline Median & 19.2 \\
\hline Range & $4.9-745.7$ \\
\hline \multicolumn{2}{|l|}{ Clinical T stage, $\mathrm{n}(\%)$} \\
\hline $\mathrm{T} 1$ & $36(47.4)$ \\
\hline $\mathrm{T} 2$ & $17(22.4)$ \\
\hline T3 & $22(28.9)$ \\
\hline $\mathrm{T} 4$ & $1(1.3)$ \\
\hline \multicolumn{2}{|l|}{ Clinical N stage, n (\%) } \\
\hline N0 & $57(75.0)$ \\
\hline N1 & $12(15.8)$ \\
\hline $\mathrm{Nx}$ & $7(9.2)$ \\
\hline \multicolumn{2}{|l|}{ Gleason score, $\mathrm{n}(\%)$} \\
\hline 6 & $3(3.9)$ \\
\hline 7 & $11(14.5)$ \\
\hline 8 & $30(39.5)$ \\
\hline 9 & $31(40.8)$ \\
\hline 10 & $1(1.3)$ \\
\hline \multicolumn{2}{|c|}{ Proportion of positive cores on biopsy, \% (NA=1) } \\
\hline Mean (SD) & $62.1(28.7)$ \\
\hline Median & 61.9 \\
\hline Range & $3.3-100.0$ \\
\hline \multicolumn{2}{|c|}{ ADT at time of the PET/CT, n (\%) (NA=4) } \\
\hline Yes & $9(11.8)$ \\
\hline No & $63(82.9)$ \\
\hline \multicolumn{2}{|c|}{ Treatment (+/- ADT), n (\%) (NA=3) } \\
\hline $\mathrm{RP}$ & 27 (35.5) \\
\hline EBRT & $35(46.1)$ \\
\hline EBRT + chemotherapy & $5(6.6)$ \\
\hline
\end{tabular}




\begin{tabular}{|l|l|}
\hline Chemotherapy & $4(5.3)$ \\
\hline Others & $2(2.6)$ \\
\hline Pathological T stage, $\mathrm{n}(\%)$ & \\
\hline T2 & $6(22.2)$ \\
\hline T3 & $21(77.8)$ \\
\hline
\end{tabular}

ADT: androgen-deprivation therapy; EBRT: external beam radiation therapy; NA: not available; PET/CT: positron-emission tomography-computed tomography; PSA: prostate-specific antigen; RP: radical prostatectomy; SD: standard deviation.

\begin{tabular}{|c|c|c|c|}
\hline Characteristics & $\begin{array}{c}\text { Positive } \\
(n=22)\end{array}$ & $\begin{array}{c}\text { Negative } \\
(n=49)\end{array}$ & $\mathbf{p}$ \\
\hline Age, years, mean (SD) & $68.9(8.5)$ & $65.4(7.8)$ & 0.096 \\
\hline $\begin{array}{l}\text { PSA at time of PET/CT, } n g / m L \text {, mean } \\
\text { (SD) }\end{array}$ & $\begin{array}{c}64.1 \\
(155.8)\end{array}$ & $24.8(22.9)$ & 0.086 \\
\hline \multicolumn{4}{|l|}{ PSA, stratified, ng/mL, n (\%) } \\
\hline PSA $\leq 20$ & $12(54.5)$ & $28(57.1)$ & 0.838 \\
\hline PSA $>20$ & $10(45.5)$ & $21(42.9)$ & \\
\hline \multicolumn{4}{|l|}{ cT stage, n (\%) } \\
\hline $\mathrm{T} 1$ & $5(22.7)$ & 29 (59.2) & \multirow[t]{3}{*}{0.005} \\
\hline $\mathrm{T} 2$ & $5(22.7)$ & $12(24.5)$ & \\
\hline T3 & $11(50.0)$ & $8(16.3)$ & \\
\hline T4 & $1(4.5)$ & $0(0.0)$ & \\
\hline \multicolumn{4}{|l|}{ cN stage, n (\%) } \\
\hline N0 & $9(40.9)$ & $43(87.8)$ & \multirow[t]{2}{*}{$<0.001$} \\
\hline N1 & $9(40.9)$ & $2(4.1)$ & \\
\hline $\mathrm{Nx}$ & $4(18.2)$ & $4(8.1)$ & \\
\hline \multicolumn{4}{|l|}{ Gleason score, $\mathrm{n}(\%)$} \\
\hline 6 & $1(4.5)$ & $2(4.1)$ & \multirow[t]{2}{*}{$<0.001$} \\
\hline 7 & $1(4.5)$ & $9(18.4)$ & \\
\hline 8 & $1(4.5)$ & $26(53.1)$ & \\
\hline 9 & $18(81.8)$ & $12(24.5)$ & \\
\hline 10 & $1(4.5)$ & $0(0.0)$ & \\
\hline $\begin{array}{l}\text { Proportion of positive cores on biopsy, } \\
\% \text { (SD) (NA=1) }\end{array}$ & $74.6(31.6)$ & $56.0(26.2)$ & 0.013 \\
\hline ADT at time of the PET/CT, n (\%) & & & \\
\hline
\end{tabular}




\begin{tabular}{|c|c|r|c|}
\hline (NA=4) & & & \\
\cline { 1 - 3 } Yes & $5(22.7)$ & $4(8.2)$ & 0.110 \\
\hline No & $17(77.3)$ & $41(83.7)$ & \\
\hline
\end{tabular}

ADT: androgen-deprivation therapy; NA: not available; PET/CT: positron-emission tomography-computed tomography; PSA: prostate-specific antigen; SD: standard deviation. 